\title{
Missed Opportunities for Prevention of Mother-to-Child Transmission of HIV (PMTCT) in Ibadan, Southwest Nigeria
}

\author{
Babatunde 0. Ogunbosi1,2*, Regina E. Oladokun1,2, Olutosin Awolude ${ }^{3,4}$, Biobele J. Brown1, \\ Olubukola A. Adeshina ${ }^{3,4}$, Modupe Kuti5 ${ }^{5}$ Babafemi Taiwo6,7, Baiba Berzins ${ }^{6,7}$, \\ Demetrious N. Kyriacou ${ }^{7,8}$, Ellen G. Chadwick ${ }^{9}$, Kikelomo Osinusi ${ }^{1,2}$, \\ Isaac F. Adewole ${ }^{3,4}$, Robert L. Murphy ${ }^{6,7}$ \\ ${ }^{1}$ Department of Paediatrics, College of Medicine, University of Ibadan, Ibadan, Nigeria \\ ${ }^{2}$ Department of Paediatrics, University College Hospital, Ibadan, Nigeria \\ ${ }^{3}$ Department of Obstetrics and Gynecology, College of Medicine, University of Ibadan, Ibadan, Nigeria \\ ${ }^{4}$ Department of Obstetrics and Gynecology, University College Hospital, Ibadan, Nigeria \\ ${ }^{5}$ Department of Chemical Pathology, College of Medicine, University of Ibadan, Ibadan, Nigeria \\ ${ }^{6}$ Department of Internal Medicine, Feinberg School of Medicine, Northwestern University, Chicago, USA \\ ${ }^{7}$ Center for Global Health, Institute for Public Health and Medicine, Feinberg School of Medicine, Northwestern \\ University, Chicago, USA \\ ${ }^{8}$ Department of Emergency Medicine, Feinberg School of Medicine, Northwestern University, Chicago, USA \\ ${ }^{9}$ Department of Pediatrics, Feinberg School of Medicine, Northwestern University, Chicago, USA \\ Email: ${ }^{*}$ tundeogunbosi@yahoo.com
}

Received 31 July 2014; revised 25 August 2014; accepted 21 September 2014

Copyright (C) 2014 by authors and Scientific Research Publishing Inc.

This work is licensed under the Creative Commons Attribution International License (CC BY).

http://creativecommons.org/licenses/by/4.0/

(c) (i) Open Access

\section{Abstract}

Background: Nigeria has the largest paediatric HIV-infected population in the world. Missed opportunities for prevention of mother-to-child transmission of HIV (PMTCT) compromise efforts at eliminating new pediatric HIV infections. Methods: Six hundred children, aged $<15$ years, presenting to the pediatric units of the University College Hospital (UCH), Ibadan Southwest Nigeria between June to December 2007 were studied. The demographics, HIV status and socioeconomic status of mothers and their children were studied. A 4-step hierarchy was used to assess the missed opportunities for PMTCT. Step 1: utilization of a health facility for antenatal care and delivery; Step 2: maternal HIV status determination during pregnancy; Step 3: provision of antiretroviral medication to HIV-infected mother and baby; and Step 4: avoidance of mixed feeding in HIV-exposed children. The rates of missed opportunities for PMTCT services at different steps in the PMTCT cascade, perinatal transmission rates, and associated factors were reported. Results:

${ }^{*}$ Corresponding author. 
There were 599 mothers and 600 children (one set of twins), 60 (10\%) were HIV infected and 56 $(93.3 \%)$ of these were adjudged perinatally infected. Of $78 \mathrm{HIV}$-infected women, 7 (9.0\%) accessed all interventions in the PMTCT cascade and $71(91.0 \%)$ had missed opportunities for PMTCT. Missed opportunities for PMTCT occurred 42.9\% in cascade Step 1, 64.2\% in Step 2, 52.6\% in step 3 and $73.7 \%$ in Step 4. All mother-baby pairs who accessed complete PMTCT interventions received care at a teaching hospital. Among infants with perinatal HIV infection, $53(94.6 \%)$ were born to mothers who had missed opportunities for PMTCT. Most women with missed opportunities attended antenatal care outside the teaching hospital setting and belonged to low socioeconomic status. Conclusion: It is imperative to expand PMTCT access to women who receive antenatal care outside the teaching hospitals and to those of low socioeconomic status.

\section{Keywords}

\section{HIV, Perinatal, Prevention, PMTCT, Missed Opportunities}

\section{Introduction}

In 2010 the World Health Organization (WHO), UNICEF and UNAIDS jointly estimated that about 34 million people were living with HIV globally, with two countries, South Africa and Nigeria, accounting for about $40 \%$ of global burden of disease [1]. In the same year an estimated 3.4 million children $<15$ years were living with HIV, $>90 \%$ in sub-Saharan Africa and over $90 \%$ acquired the virus perinatally [1]. The WHO and the Office of the United States Global AIDS Coordinator are committed to eliminating new pediatric HIV infections by 2015 and keeping their mothers healthy [2]. Without any intervention, mother-to-child transmission (MTCT) of HIV ranges from $14 \%$ - 32\% in non-breastfeeding (mostly industrialized) populations to $25 \%$ - $48 \%$ in breastfeeding (mostly resource limited) populations [3] [4]. Combinations of newer antiretroviral agents and targeted elective caesarean section have been found to reduce MTCT rates to less than $2 \%$ in non-breastfeeding populations and less than $5 \%$ in breastfeeding populations [5]-[12].

Globally, Nigeria has the second largest burden of HIV with 3.3 million people infected in 2009, and the largest pediatric HIV-infected population in the world, with over 360,000 children aged $<15$ years living with HIV [13]. Of the 22 countries with the highest burden of HIV infection, a decline in MTCT rates was observed from 2009 to 2010 except in Nigeria where the rates remain unchanged at 33\% and Mozambique where a slight increase was observed from $29 \%$ to $31 \%$. During this period Nigeria had an estimated 75,000 new pediatric HIV infections, the highest in the world [1] .

To prevent perinatal HIV infection, a pregnant woman with HIV infection needs to be identified early in pregnancy and ARVs administered to mother and baby, including the breastfeeding period where applicable. Missed opportunities for prevention of mother-to-child transmission of HIV (PMTCT) occur when any of these activities do not take place and factors, which vary in different geographical and cultural settings, influence this [14]-[17].

In recent years, the Nigerian government, and her development partners, have made immense investments in providing PMTCT services. In the face of the challenging burden of paediatric HIV, missed opportunities in PMTCT will further compromise any effort to meet the daunting task of eliminating new pediatric HIV infections in Nigeria. This study investigated steps in the PMTCT cascade where missed opportunities for PMTCT occurred among mothers of the children attending a pediatric unit in Ibadan, southwest Nigeria and the contributing factors. It also determined the magnitude and effect of missed opportunities for PMTCT on perinatal HIV and possible areas for intervention to improve PMTCT outcomes.

\section{Methods}

This is a secondary analysis of data collected from a previously published prospective cross-sectional study, conducted between June to December 2007, to evaluate the prevalence and clinical pattern of pediatric HIV infection at the University College Hospital Ibadan, Southwest Nigeria [18]. The database contained information on 600 consecutively enrolled children aged less than 15years presenting to the pediatric unit of the University College Hospital (UCH), Ibadan, Nigeria for the first time during the study period irrespective of the reason for 
presentation. The local standard of care during the study period was based on the 2006 WHO recommendations which preceded the 2010 guidelines [19].

Collected data included the child's age, sex, date of birth, symptoms and signs, anthropometry, place of delivery, mode of delivery, breast feeding option practiced, history of sexual exposure, blood transfusion, result of rapid HIV test, confirmatory test result (HIV DNA PCR if $<18$ months, Western Blot if $\geq 18$ months), ART used for PMTCT. Also included in the database was information on mother's age, educational status, occupation, place of antenatal care, if she was tested for HIV in pregnancy, her HIV status in pregnancy, ART used for PMTCT, result of provider initiated HIV counseling and testing (a subset of mothers who gave consent were screened in the course of the study).

A modified 4-step hierarchy for reducing the odds for perinatal HIV transmission recommended in 1999 by the Institute of Medicine [20] and as described by Peters et al. [14] was used to assess the contribution of the different steps in the PMTCT cascade to perinatal HIV: Step 1: utilization of a health facility for antenatal care and delivery; Step 2: maternal HIV status known in pregnancy, i.e. pregnant woman is screened for HIV infection and the result communicated to her; Step 3: maternal ART for PMTCT and neonatal ART; and Step 4: practice of exclusive breastfeeding or exclusive replacement feeding, i.e. avoidance of mixed feeding as this increases the risk of MTCT of HIV. Complete PMTCT occurred if mother-baby pair accessed all 4 steps in the cascade and missed opportunity for PMTCT occurred if one or more of the 4 steps in the cascade did not occur.

The primary end point was the rate of MTCT of HIV among mothers who accessed complete PMTCT services compared to those who had missed opportunities for PMTCT services. We also analyzed rates of access to interventions at the various steps in the PMTCT cascade and the factors associated with missed opportunities in the cascade.

Continuous variables are reported as means with standard deviations or medians, while categorical variables are reported as proportions. Univariate analysis was used to evaluate factors associated with missed opportunities for PMTCT and multivariate regression analysis was used to determine factors that are predictive of missed opportunities for PMTCT. Odds ratios and 95\% confidence intervals are reported and p-value $<0.05$ were considered statistically significant. Data was analyzed using SPSS 11.0 for Windows Chicago USA [21] and STATA version 10, StataCorp LP TX, USA. The University of Ibadan/University College Hospital Ibadan Ethics Committee and the Northwestern University Institutional Review Board approved the study.

\section{Results}

Of 600 children enrolled from 599 mothers (one set of twins), 329 (54.9\%) were males, 271 (45.1\%) were females and their ages ranged from one day to 179 months with a median age of 16 months. The HIV prevalence among the children was 10\% (60/600), and 56 (93.3\%) of the HIV-infected children acquired the infection via mother-to-child transmission. Sexual and blood transfusion related modes of transmission accounted for 2 (3.33\%) each. These non-perinatally infected children and their mothers were excluded from further analysis for this study. The prevalence and clinical pattern of HIV infection in this cohort has been published elsewhere [18].

The mean age of the 595 mothers was $31.06( \pm 6.5)$ years, 213 (35.8\%) had been tested for HIV in pregnancy and knew their HIV status. Of these, 19 (3.2\%) were HIV-infected and provided proof in the form of a HIV test result or evidence of antiretroviral usage and 194 (32.6\%) reported they were HIV-uninfected. In 382 (64.2\%) women, their HIV status was unknown, either because they were not tested in pregnancy, did not receive their test result or were not available due to demise or had travelled during the study period. A subset of 351 women had provider-initiated-HIV testing and counseling (PITC) testing; all those who reported being HIV-uninfected in pregnancy and 157 with unknown HIV status. Of these, 59 were confirmed HIV-infected and 292 HIV-uninfected (Figure 1). In all 78 (13.1\%) women were confirmed HIV-infected, 292 (49.1\%) were confirmed HIVuninfected and in 225 (37.8\%) women, their HIV status remained unknown as they were deceased, declined testing or were not present at the study site during study period (Figure 1). Among children of the women who reported being HIV-infected in pregnancy 8 were HIV-infected. In women who said they were not HIV-infected in pregnancy, 13 of their children were HIV-infected and those with unknown HIV status in pregnancy had 39 children infected with HIV (Table 1).

Among the 78 women with HIV infection, 7(9.0\%) women accessed all interventions in the PMTCT cascade and $71(91.0 \%)$ had missed opportunities for PMTCT. Of the 56 infants with perinatal HIV infection, 3 were born to women who had complete PMTCT interventions and 53(94.6\%) were born to mothers who did not access one or more steps in the PMTCT cascade and thus had missed opportunities for PMTCT. A summary of access to PMTCT interventions and infants HIV status is presented in Table 2. 


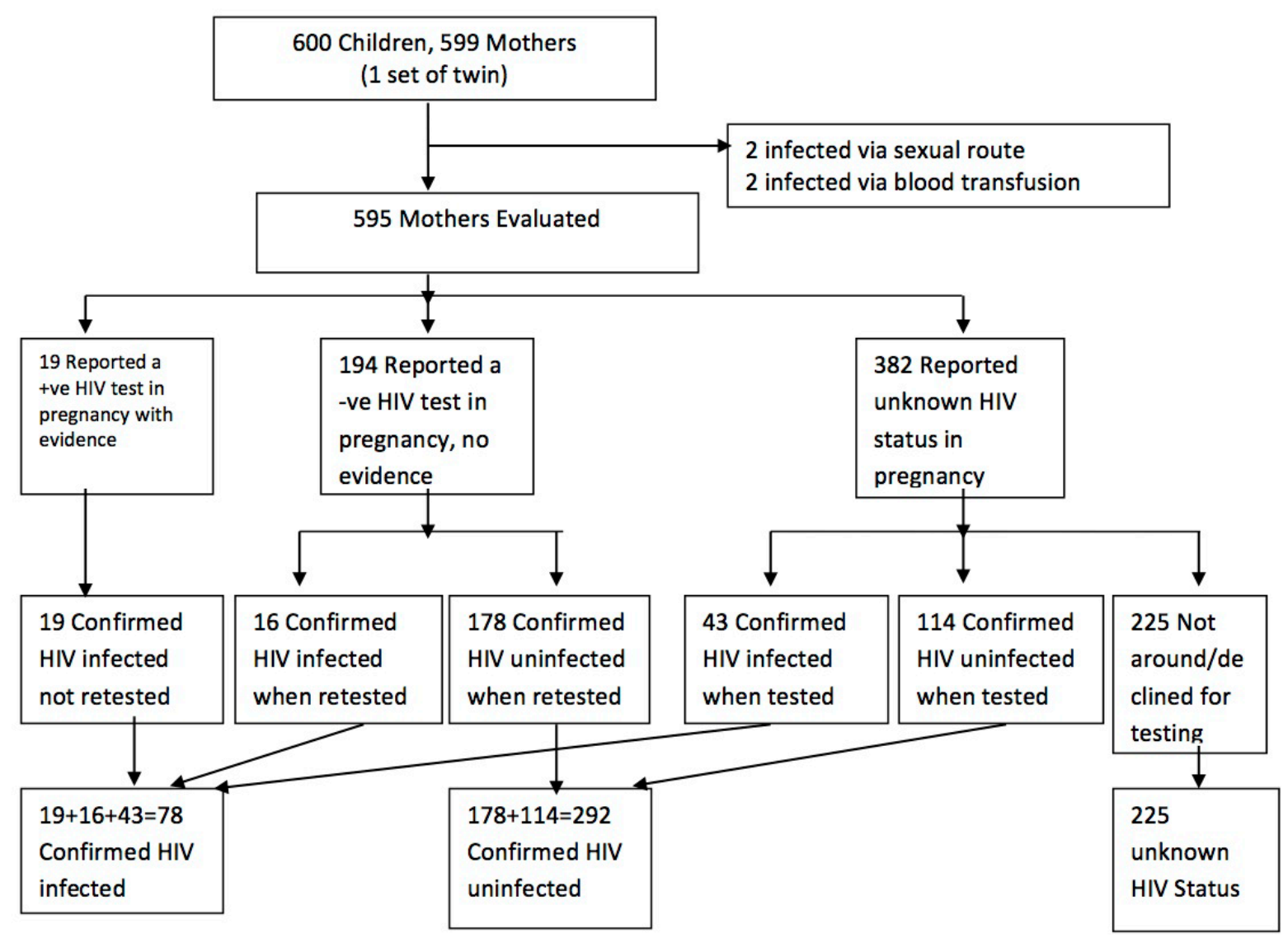

Figure 1. Summary of maternal HIV status.

Table 1. Summary of mother's reported HIV status in pregnancy and child’s HIV status after testing.

\begin{tabular}{cccc}
\hline $\begin{array}{c}\text { Mother's Reported HIV Status in } \\
\text { Pregnancy }\end{array}$ & N (\%) & \multicolumn{2}{c}{ HIV Status of Child } \\
\cline { 3 - 3 } HIV Infected & $19(3.2)$ & $8(14.3)$ & Negative N (\%) \\
HIV Uninfected & $194(32.6)$ & $13(23.2)$ & $182(33.7)$ \\
Unknown HIV Status & $382(64.2)$ & $35(62.5)$ & $347(64.3)$ \\
Total & 595 & $56(100)$ & 540 \\
\hline
\end{tabular}

Table 2. Summary of access to PMTCT interventions and infant HIV status of the 78 mothers with HIV infection.

\begin{tabular}{cccc}
\hline \multirow{2}{*}{ PMTCT Access } & \multicolumn{2}{c}{ Infant HIV Status N (\%) } & \multirow{2}{*}{ Total N (\%) } \\
\cline { 2 - 3 } Accessed complete PMTCT interventions & Positive & Negative & $7(8.9)$ \\
Missed opportunities for PMTCT & $3(5.4)$ & $4(18.2)$ & $71(91.0)$ \\
Total & $53(94.6)$ & $18(81.8)$ & 78 \\
\hline
\end{tabular}

Missed opportunities occurred at all the four steps in the PMTCT cascade. In Step 1, 42.9\% (340/595) of the mothers did not have formal antenatal care; in Step 2, 64.2\% of the mothers did not have knowledge of their HIV status in pregnancy. In addition, $52.6 \%$ of both mothers who had a knowledge of their positive HIV status 
and their babies did not receive antiretroviral medications for their own health or PMTCT in Step 3 and 73.7\% of the babies had mixed breastfeeding in Step 4 (Figure 2).

Most women who did not have knowledge of their HIV status accessed ANC at private hospitals and belonged to the lower socioeconomic classes (Table 3 and Figure 3), the sample size was however not large enough to do a regression analysis. All mother-baby pairs who accessed complete PMTCT interventions received care at a tertiary hospital.

\section{Discussion}

In this study, 91\% of eligible mothers had missed opportunities for PMTCT and the majority transmitted HIV infection to their children. Missed opportunities occurred at every step in the PMTCT cascade in a large proportion of the women studied, and most were of low socioeconomic status. Complete access to PMTCT intervene-

\section{Step 1}

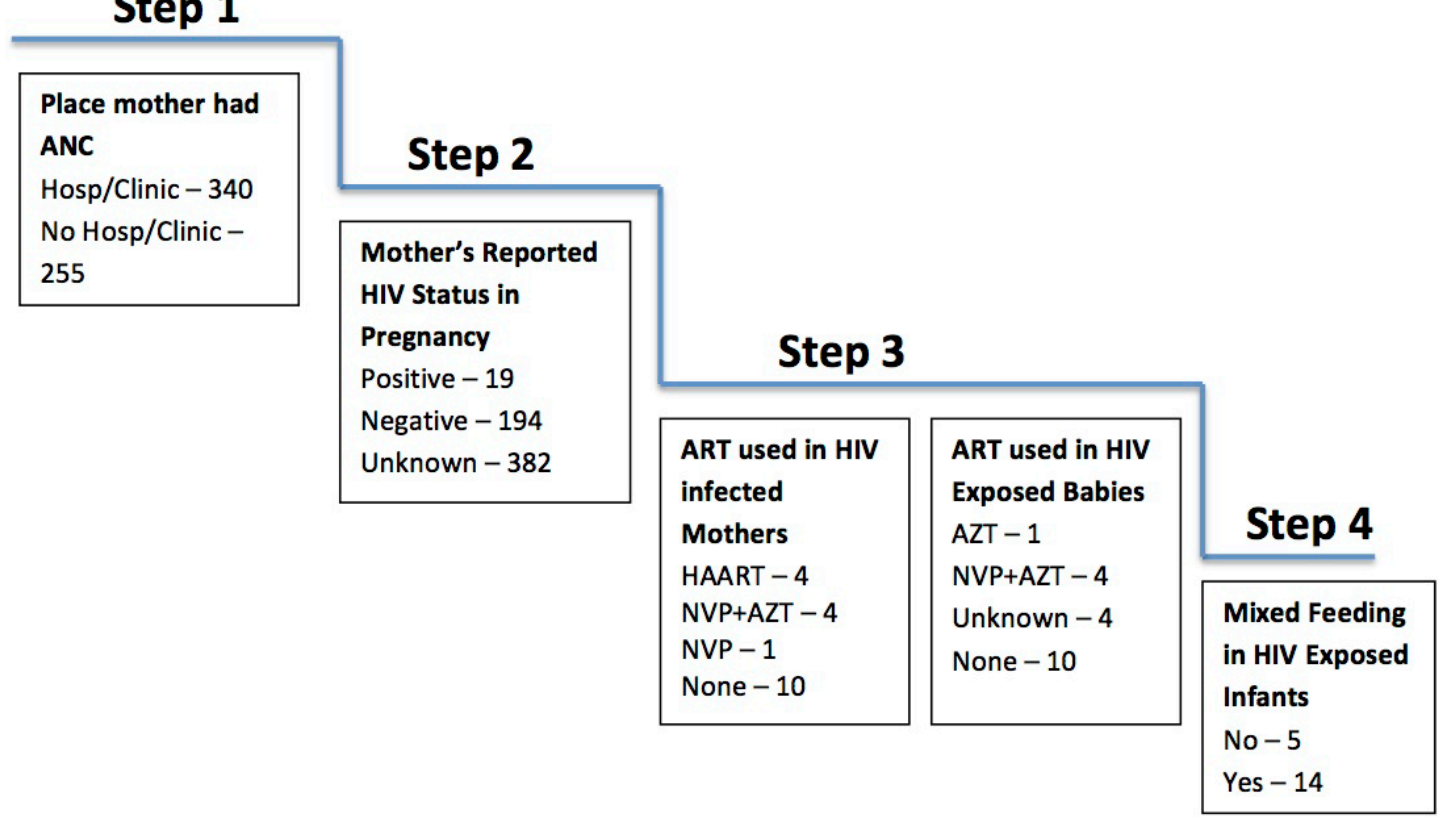

Figure 2. Utilization of intervention in the PMTCT cascade.

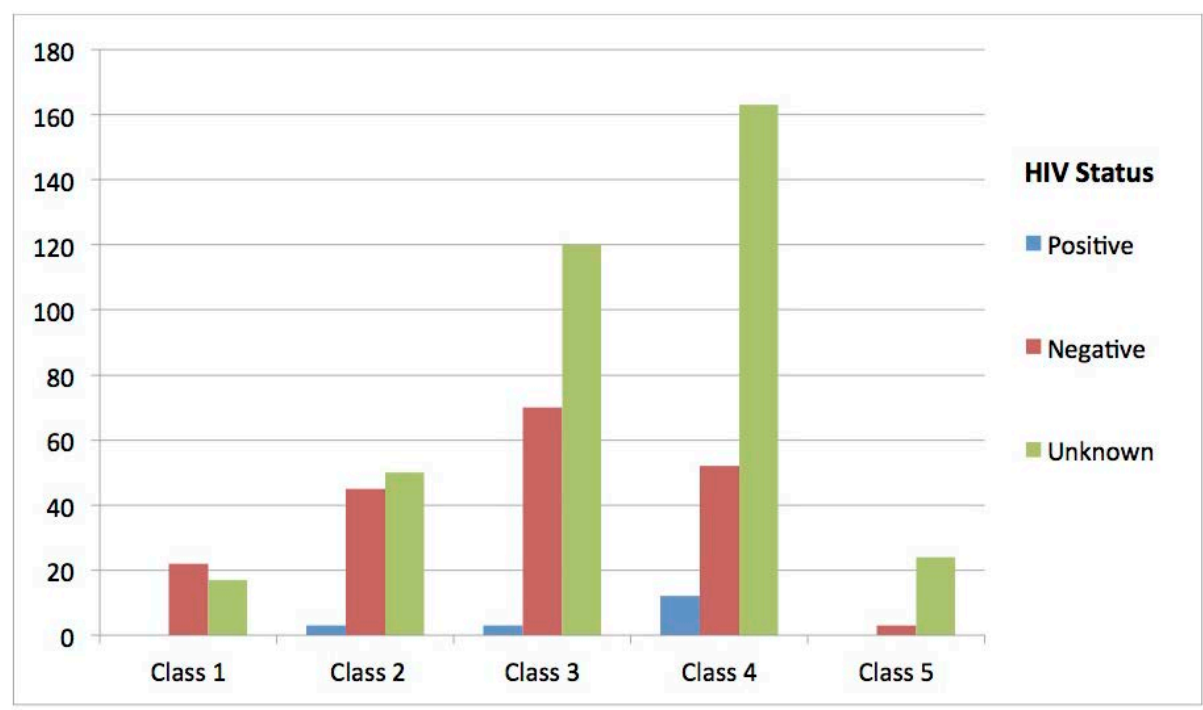

Figure 3. Socioeconnmic class and mother’s HIV status in pregnancy. 
Table 3. Place of ANC and mother's reported HIV status in pregnancy.

\begin{tabular}{ccccc}
\hline Place of Delivery/ANC & \multicolumn{2}{c}{ Mother's Reported HIV Status in Pregnancy N (\%) } & $\begin{array}{c}\text { Total } \\
\text { N (\%) }\end{array}$ \\
\cline { 2 - 4 } Government PHC & Positive & Negative & Unknown & $22(3.7)$ \\
Gen Hospital & $0(0)$ & $5(2.6)$ & $17(4.5)$ & $56(9.4)$ \\
Teaching Hospital & $1(5.3)$ & $16(8.2)$ & $39(10.2)$ & $54(9.1)$ \\
Private Hospital & $10(52.6)$ & $26(13.4)$ & $18(4.7)$ & $111(29.1)$ \\
Mission Hospital & $2(10.5)$ & $45(23.2)$ & $36(9.4)$ & $50(8.4)$ \\
Faith Based Maternity Center & $0(0)$ & $14(7.2)$ & $18(4.7)$ & $24(4.0)$ \\
At home & $0(0)$ & $6(3.1)$ & $30(7.9)$ & $38(6.4)$ \\
Non-Faith Based Maternity Center & $3(15.8)$ & $5(2.6)$ & $4(1.0)$ & $5(0.8)$ \\
Others & $0(0)$ & $1(0.5)$ & $2(0.5)$ & $3(0.5)$ \\
Not Known & $0(0)$ & $1(0.5)$ & $107(28.0)$ & $185(31.1)$ \\
Total & $3(15.8)$ & $75(38.7)$ & $\mathbf{3 8 2}$ & $\mathbf{5 9 5}$ \\
\hline
\end{tabular}

tions was observed only among women who received antenatal care in tertiary hospitals.

Achieving the goal of eliminating new perinatal HIV infections by 2015 will require every pregnant woman to access all interventions in the PMTCT cascade. However, this is not always possible and missed opportunities have been observed in both developed countries with well established health systems and ready access to these interventions, as well as in developing countries. In the US where implementation of PMTCT methods has almost achieved elimination of new pediatric HIV infections, Peters et al. analyzed data on 4755 infants born to HIV-infected mothers from 6 US sites between 1994-2000 as part of the CDC's Pediatric Spectrum of HIV Disease Project and found that 56\% of mothers of HIV-infected infants compared to $16 \%$ of HIV uninfected infants had missed opportunities for PMTCT [14]. Similarly in 2011, the European Collaborative Study reported a MTCT rate of $7.4 \%$ among HIV positive women who received insufficient ART for PMTCT compared to $1.1 \%$ among those who had $\geq 14$ days of antenatal ART [17]. In resource poor countries where health systems are less well developed and women have limited access to interventions for PMTCT, the effect of missed opportunities can be profound as demonstrated in this study, and similar to the report by Turchi et al. from Brazil [15].

The entry point for PMTCT is accessing antenatal care (ANC) at health facilities where the HIV status of the woman can be determined during pregnancy. A significant proportion of women in this study did not have supervised care in pregnancy at hospitals or clinics, the place where HIV testing is often done in Nigeria. This is a recognized problem in Nigeria where in 2008 only an estimated 58\% of pregnant women accessed ANC services provided by skilled health personnel and 35\% had health facility delivery [22]. Less than $25 \%$ of pregnant women had access to Voluntary Counseling and Testing (VCT) for HIV in the same year [22]. Recent analysis of data from the Nigeria Demographic and Health Survey 2008 showed that low socioeconomic status is the most important factor for low utilization of antenatal and other maternal care services [23]. This is not unconnected with the relative high cost of health service for most of Nigeria's poor populace and the mostly out of pocket services that operates in most health facilities in Nigeria. Therefore, devising low-cost innovative ways to reach this group of women is a major task that must be accomplished in any intervention to reduce missed opportunities for PMTCT.

As observed by Stringer et al. [24] in the PEARL study and Oladokun et al. [25] in Nigeria, mother-baby pairs in developing countries may not navigate the PMTCT cascade successfully even when they have access to antenatal care. This was again demonstrated in this study among the women who accessed antenatal care in hospitals or clinics where HIV screening should have been available. Some women were not screened for HIV infection during pregnancy or did not collect their HIV test results thus their HIV status was not determined in pregnancy. Most of these women accessed care in private hospitals and were from the lower socioeconomic classes. HIV testing is not readily available in most private hospitals in Nigeria and where available, the cost is 
prohibitive for most women, and usually out of pocket expenses. For some women who reported they were HIVuninfected, repeat testing revealed they were HIV-infected. This is not uncommon as some women acquire infection later in pregnancy or after delivery underscoring the need for a repeat testing late in pregnancy as has been advocated.

Administration of antiretroviral medication is the most important intervention in the PMTCT cascade and can reduce perinatal transmission by more than 60\% [5] [6]. In HIV infected pregnant women who were identified late in pregnancy or in labor, administering antiretroviral medications to reduce perinatal HIV transmission remains efficacious [26]. Nigeria accounts for about 32\% of the gap in reaching the WHO global target of $80 \%$ coverage of antiretroviral medications for PMTCT [27] and most PMTCT programs are in tertiary or secondary health facilities, financially and geographically out of reach of most women in need of this therapy. In the limited number of women who knew they were HIV-infected during pregnancy in this study, most did not access antiretroviral medications for themselves or their infants for the prevention of vertical transmission. The majority of those who received antiretroviral medications received antenatal care in tertiary hospitals where PMTCT programs are well established. In Brazil, Turchi et al. [15] had identified not being cared for by a specialist and place of delivery as factors associated with missed opportunities for PMTCT. This suggests a need to decentralize PMTCT services in developing countries to increase access to antiretroviral medications for PMTCT.

Mixed feeding, the addition of supplemental feeds to breastfeeding increases the risk for HIV transmission via breast milk. The World Health Organization (WHO) previously recommended the avoidance of mixed feeding among HIV infected women in breastfeeding populations [28], which was fraught with cultural challenges in most developing countries; thus high rates of mixed feeding persisted as seen in this study. The WHO [19], in recognition of this challenge, has revised this recommendation; this was introduced in Nigeria in 2010, but effect of this is yet to be seen.

This study has some limitations being a secondary analysis of information collected in an observational cross-sectional study. Most of the information regarding access to interventions for PMTCT was based on the women's recall or the report of a care-giver which might be inaccurate and difficult to validate, as already observed by Stringer et al. [24]. The sample size of this study does not allow for detection of significant differences or full exploration of factors contributing to missed opportunities for PMTCT. However, our findings highlight the fact that missed opportunities for PMTCT remain a significant problem in Nigeria and this promotes perinatal HIV transmission. Further prospective studies are needed to confirm these findings and explore the contributing factors.

\section{Conclusion}

The vast majority of newborn infants in our study with perinatal acquisition of HIV were born to mothers who had the opportunity for PMTCT. Nigeria needs to expand PMTCT access, especially to women who receive antenatal care outside the teaching hospitals and design interventions that will reach women of low socioeconomic status.

\section{Competing Interests}

No competing interest declared by the authors.

\section{Authors' Contributions}

BOO conceived the idea, collected and analyzed data, wrote the initial and final drafts of the paper. REO and KO supported BOO in data collection and analysis and contributed to initial and final drafts. OA, OAA and MK contributed to data analysis and initial and final drafts of the paper.EGC, DK, BT, BB, IFA and RLM all contributed to conceiving the idea, study design, data analysis and contributed to initial and final drafts of the paper.

\section{Acknowledgements}

The project described was supported by Award Number 5D43TW007995 (Northwestern University AIDS International Training and Research Program) and educational training was supported by Award Number 1R24TW008878 (Medical Education Partnership Initiative Nigeria) from the Fogarty International Center. The content is solely the responsibility of the authors and does not necessarily represent the official views of the Fogarty International 
Center or the National Institutes of Health.

\section{References}

[1] UNAIDS (2011) Global HIV/AIDS Response-Epidemic Update and Health Sector Progress towards Universal Access-Progress Report 2011. UNAIDS, Geneva.

[2] UNAIDS (2011) Global Plan towards the Elimination of New HIV Infections among Children by 2015 and Keeping Their Mothers Alive 2011-2015. UNAIDS, Geneva.

[3] De Cock, K.M., Fowler, M.G., Mercier, E., de Vincenzi, I., Saba, J., Hoff, E., Alnwick, D.J., Rogers, M. and Shaffer, N. (2000) Prevention of Mother-to-Child HIV Transmission in Resource-Poor Countries: Translating Research into Policy and Practice. JAMA, 283, 1175-1182. http://dx.doi.org/10.1001/jama.283.9.1175

[4] Wiktor, S.Z., Ekpini, E. and Nduati, R.W. (1997) Prevention of Mother-to-Child Transmission of HIV-1 in Africa. AIDS, 11, S79-S87.

[5] Connor, E.M., Sperling, R.S., Gelber, R., Kiselev, P., Scott, G., O’Sullivan, M.J., Van Dyke, R., Bey, M., Shearer, W., Jacobson, R.L., et al. (1994) Reduction of Maternal-Infant Transmission of Human Immunodeficiency Virus Type 1 with Zidovudine Treatment. Pediatric AIDS Clinical Trials Group Protocol 076 Study Group. The New England Journal of Medicine, 331, 1173-1180. http://dx.doi.org/10.1056/NEJM199411033311801

[6] Connor, E.M. and Mofenson, L.M. (1995) Zidovudine for the Reduction of Perinatal Human Immunodeficiency Virus Transmission: Pediatric AIDS Clinical Trials Group Protocol 076-Results and Treatment Recommendations. The Pediatric Infectious Disease Journal, 14, 536-541. http://dx.doi.org/10.1097/00006454-199506000-00012

[7] Brocklehurst, P. and Volmink, J. (2002) Antiretrovirals for Reducing the Risk of Mother-to-Child Transmission of HIV Infection. The Cochrane Database of Systematic Reviews 2002, CD003510.

[8] Mandelbrot, L., Landreau-Mascaro, A., Rekacewicz, C., Berrebi, A., Benifla, J.L., Burgard, M., Lachassine, E., Barret, B., Chaix, M.L., Bongain, A., et al. (2001) Lamivudine-Zidovudine Combination for Prevention of Maternal-Infant Transmission of HIV-1. JAMA: The Journal of the American Medical Association, 285, 2083-2093. http://dx.doi.org/10.1001/jama.285.16.2083

[9] Brocklehurst, P. and Volmink, J. (2002) Antiretrovirals for Reducing the Risk of Mother-to-Child Transmission of HIV Infection. The Cochrane Database of Systematic Reviews 2002, CD003510.

[10] The European Mode of Delivery Collaboration (1999) Elective Caesarean-Section versus Vaginal Delivery in Prevention of Vertical HIV-1 Transmission: A Randomised Clinical Trial. Lancet, 353, 1035-1039. http://dx.doi.org/10.1016/S0140-6736(98)08084-2

[11] Brocklehurst, P. (2002) Interventions for Reducing the Risk of Mother-to-Child Transmission of HIV Infection. The Cochrane Database of Systematic Reviews, Published Online.

[12] Mofenson, L.M. (2010) Prevention in Neglected Subpopulations: Prevention of Mother-to-Child Transmission of HIV Infection. Clinical Infectious Diseases, 50, S130-148. http://dx.doi.org/10.1086/651484

[13] UNAIDS (2010) Global Report: UNAIDS Report on the Global AIDS Epidemic 2010. UNAIDS, Geneva.

[14] Peters, V., Liu, K.L., Dominguez, K., Frederick, T., Melville, S., Hsu, H.W., Ortiz, I., Rakusan, T., Gill, B. and Thomas, P. (2003) Missed Opportunities for Perinatal HIV Prevention among HIV-Exposed Infants Born 1996-2000, Pediatric Spectrum of HIV Disease Cohort. Pediatrics, 111, 1186-1191.

[15] Turchi, M.D., Duarte, S. and Martelli, C.M. (2007) Mother-to-Child Transmission of HIV: Risk Factors and Missed Opportunities for Prevention among Pregnant Women Attending Health Services in Goiânia, Goiás State, Brazil. Cadernos de Saúde Pública, 23, S390-S401. http://dx.doi.org/10.1590/S0102-311X2007001500007

[16] Rodrigues, C.S., Guimarães, M.D. and César, C.C. (2008) Missed Opportunities for Congenital Syphilis and HIV Perinatal Transmission Prevention. Revista de Saúde Pública, 42, 851-858. http://dx.doi.org/10.1590/S0034-89102008000500010

[17] Bailey, H., Townsend, C., Cortina-Borja, M. and Thorne, C. (2011) Insufficient Antiretroviral Therapy in Pregnancy: Missed Opportunities for Prevention of Mother-to-Child Transmission of HIV in Europe. Antiviral Therapy, 16, 895903. http://dx.doi.org/10.3851/IMP1849

[18] Ogunbosi, B.O., Oladokun, R.E., Brown, B.J. and Osinusi, K.I. (2011) Prevalence and Clinical Pattern of Paediatric HIV Infection at the University College Hospital, Ibadan, Nigeria: A Prospective Cross-Sectional Study. Italian Journal of Pediatrics, 37, 29.

[19] WHO (2010) Antiretroviral Drugs for Treating Pregnant Women and Preventing HIV Infection in Infants: Recommendations for a Public Health Approach. 2010 Version. WHO, Geneva.

[20] Reducing the Odds (1999) Preventing Perinatal Transmission of HIV in the US, US Institute of Medicine Report. Institute of Medicine, Washington DC. 
[21] SPSS 11.0 for Windows (2001) SPSS Inc., Chicago.

[22] NPC (2009) Nigeria Demographic and Health Survey 2008. National Population Commission, Abuja.

[23] Rai, R.K., Singh, P.K. and Singh, L. (2012) Utilization of Maternal Health Care Services among Married Adolescent Women: Insights from the Nigeria Demographic and Health Survey, 2008. Women's Health Issues, 22, e407-414. http://dx.doi.org/10.1016/j.whi.2012.05.001

[24] Stringer, E.M., Ekouevi, D.K., Coetzee, D., Tih, P.M., Creek, T.L., Stinson, K., Giganti, M.J., Welty, T.K., Chintu, N., Chi, B.H., et al. (2010) Coverage of Nevirapine-Based Services to Prevent Mother-to-Child HIV Transmission in 4 African Countries. JAMA, 304, 293-302. http://dx.doi.org/10.1001/jama.2010.990

[25] Oladokun, R.E., Awolude, O., Brown, B.J., Adesina, O., Oladokun, A., Roberts, A., Odaibo, G., Osinusi, K., Olaleye, D., Adewole, I.F., et al. (2010) Service Uptake and Performance of the Prevention of Mother-to-Child Transmission (PMTCT) Programme in Ibadan, Nigeria. African Journal of Medicine and Medical Sciences, 39, 81-87.

[26] Wade, N.A., Birkhead, G.S., Warren, B.L., Charbonneau, T.T., French, P.T., Wang, L., Baum, J.B., Tesoriero, J.M. and Savicki, R. (1998) Abbreviated Regimens of Zidovudine Prophylaxis and Perinatal Transmission of the Human Immunodeficiency Virus. The New England Journal of Medicine, 339, 1409-1414. http://dx.doi.org/10.1056/NEJM199811123392001

[27] UNAIDS (2009) Towards Universal Access: Scaling up Priority HIV/AIDS Interventions in the Health Sector. Progress Report 2009.

[28] WHO (2006) Antiretroviral Drugs for Treating Pregnant Women and Preventing HIV Infection in Infants: Towards Universal Access: Recommendations for a Public Health Approach. 2006 Version. WHO, Geneva. 
Scientific Research Publishing (SCIRP) is one of the largest Open Access journal publishers. It is currently publishing more than 200 open access, online, peer-reviewed journals covering a wide range of academic disciplines. SCIRP serves the worldwide academic communities and contributes to the progress and application of science with its publication.

Other selected journals from SCIRP are listed as below. Submit your manuscript to us via either submit@scirp.org or Online Submission Portal.
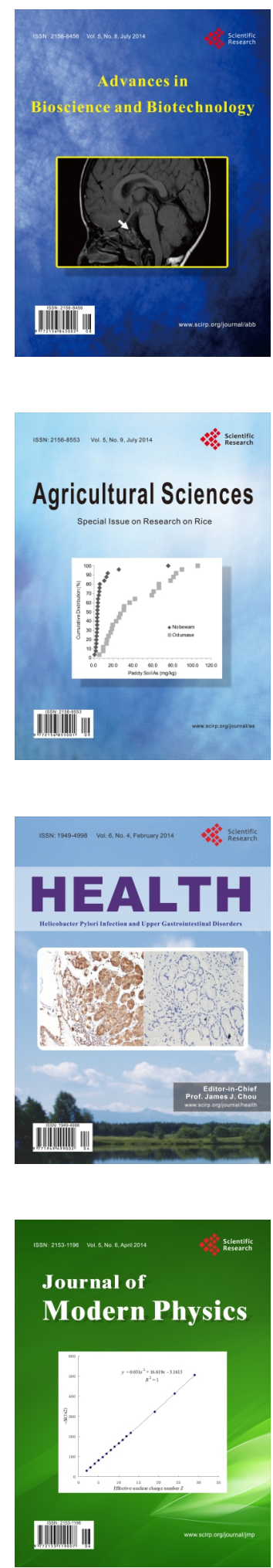
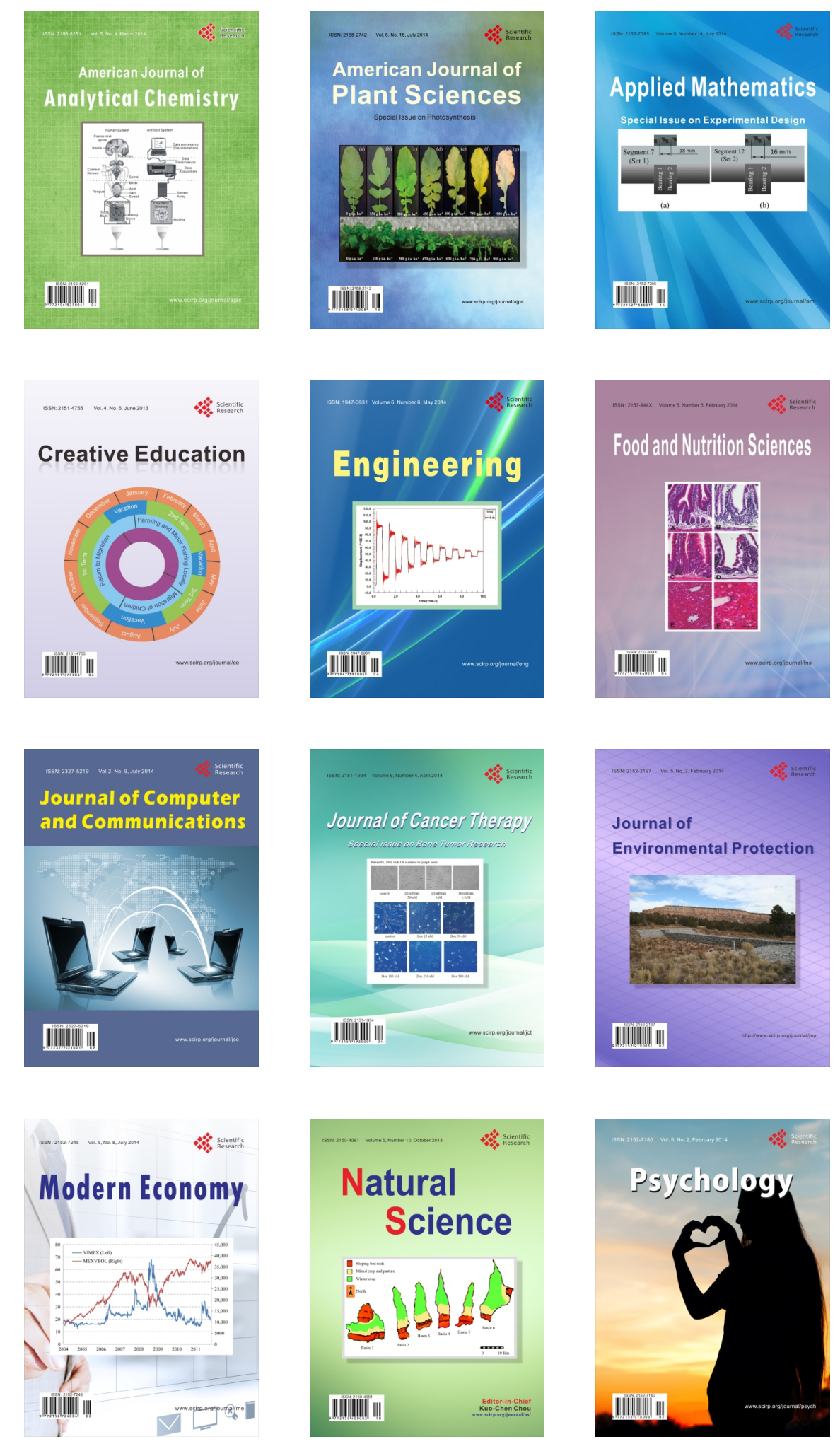